\title{
ENDOMETRIOSE VESICAL: ASPECTOS DIAGNÓSTICOS E TERAPÊUTICOS
}

*M. Tobias-Machado, R. DI Giuseppe, C.P. Barbosa, M. Borrelli, E.R. Wroclawski

TrabalhorealizadopelasDisciplinas de Urologiae GinecologiadaFaculdadedeMedicinadoABC(FMABC)

UniterMos: Endometriose. Cirurgia endoscópica. Doenças do trato urinário.

KEY wORDS: Endometriosis. Endoscopic surgery. Urinary tract diseases.

\section{INTRODUÇÃO}

Endometriose é a presença de tecido endometrial ectópico e/ou estroma fora da cavidade uterina. Foi observada, pela primeira vez, por Rokitansky (1869) em material de necrópsia. Mais tarde, Sampson (1927) definiu a endometriose como um achado de tecido com aspectos histológicos e funcionais semelhantes ao endométrio tópico, em outros locais.

A endometriose é a segunda afecção cirúrgica ginecológica, perdendo apenas para a miomatose uterina. Nos EUA é a terceira causa de internação e a causa mais comum de histerectomia!.

Nos últimos anos têm-se verificado um aumento da freqüência de endometriose. Esse aumento se deve às mudanças de hábitos femininos; aumento da idade da primeira gestação, maior intervalo entre as gestações, o que promove maior tempo de exposição estrogênica e maior freqüência de menstruações.

A endometriose pode causar alteração da fertilidade, dor pélvica ou até ser assintomática. Possui estágios diferentes de evolução, podendo se apresentar de forma mínima, leve, moderada ou grave. As for-

*Correspondência:

R. OscarFreire, 1546-apto. 56-Cep:05409-010 Cerqueira César-SãoPaulo-Brasil-Tel.: 3081-8674 E-mail:hcor@ncor.com.br mas mínimas e leves podem se agravar com o passar do tempo 2,3,4. Além disso, a afecção tem sua gravidade relacionada com a freqüência, quantidade e duração das menstruações.

\section{Etiopatogenia}

Teoria do implante endometrial explica o aparecimento da endometriose em cicatriz de cesáreas ou após histerectomias.

Teoria da metaplasia celômica justifica o desenvolvimento da endometriose em muIheres com agenesia uterina.

A disseminacão por via hematogênica justifica as formas extraperitoneais.

A teoria do refluxo tubário do fluido menstrual ocorre com maior freqüência nos locais onde o fluido menstrual se deposita 5 .

Alguns autores sugerem a participação do sistema autoimune ${ }^{6,7,8}$. Acreditase na diminuição da atividade de linfócitos denominados "natural killers" que agiriam contra antígenos endometriais autó logos. A diminuição da atividade dessas células estaria relacionada com a gravidade da doença?.

Recentemente, estudou-se a possibilidade de que a endometriose peritoneal e os nódulos endometrióticos no septo retovaginal fossem duas entidades distintas e foi proposto designá-las de adenomiose reto-

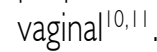

\begin{tabular}{cc}
\hline $\begin{array}{c}\text { Tabela I - Incidência de endometriose do } \\
\text { trato urinário (adaptado de 20) }\end{array}$ \\
\hline Localização & Número de casos (\%) \\
Rim & $6(4,0)$ \\
Ureter & $\mid 5(9,9)$ \\
Bexiga & $\mid 27(84,1)$ \\
Uretra & $2(2,0)$ \\
Total & $|5|(100)$ \\
\hline
\end{tabular}

\section{INCIDÊNCIA}

A endometriose incide em 10\% das mulheres entre 25 a 40 anos de idade. Acomete em até $50 \%$ mulheres inférteis ${ }^{12-16} \mathrm{e}$ possui sua maior freqüência em nulíparas. Em assintomáticas pode incidir de $6 \%{ }^{17}$ até $43 \%{ }^{18}$.

Os locais mais acometidos são o fundo de saco de Douglas, ovários, ligamento redondo, tubas uterinas, cérvix, vagina.

Apenas I a $2 \%$ dos casos de endometriose acomete o trato urinário, sendo que a bexiga é acometida em $84 \%$ dos $\operatorname{casos}^{19}$ e, em seguida, o ureter. Na bexiga, as regiões mais freqüentemente acometidas são o trígono e o colo vesical. A tabela I mostra a freqüência de endometriose de trato urinário.

Como diagnóstico diferencial da endometriose vesical temos: angiomas, papilomas, inflamação ou úlcera vesical localizada e carcinoma infiltrativo. 


\section{Quadro Clínico}

A natureza e a severidade dos sintomas vesicais variam conforme a localização, tamanho e duração do tumor (tabela 2).

O mais comum a ser encontrado é algia e desconforto supra púbico ou na região vésico-vaginal (78\%), além de disúria, polaciúria, urgência miccional ${ }^{19}$.

A hematúria cíclica é encontrada em $25 \%$ dos casos, enquanto que distúrbios menstruais (dismenorréia, menorragia, metrorragia) foram encontrados em $50 \%$ dos casos $^{20}$.

Em 40\% das pacientes encontrou-se uma massa palpável ao toque vaginal.

\section{Exames Subsidiários}

Tanto no estudo ultra-sonográfico quanto na tomografia computadorizada e ressonância magnética, o achado mais comum é vegetação endoluminal na parede vesical. A urografia excretora pode revelar falha de enchimento vesical ou hidronefrose se houver comprometimento ureteral' ${ }^{21}$ (tabela 3).

A laparoscopia diagnóstica, realizada em casos de algia pélvica, pode localizar as lesões, inspecionar os órgãos pélvicos e toda a cavidade peritoneal permitindo a realização de biópsias.

A cistoscopia é o exame de preferência e seus achados variam conforme a fase do ciclo menstrual. Dessa forma, há necessidade de várias cistoscopias em diferentes fases do ciclo menstrual para um diagnóstico mais apurado $^{20}$. Deve-se ressaltar, ainda, que 0 diagnóstico deve ser realizado através de biópsia profunda da parede vesical, geralmente conseguido através de ressecção com alça, pois biópsias superficiais podem revelar apenas cistites inespecíficas.

Período menstrual: pode ser detectado uma área elevada na parede vesical posterior, perto do trígono ou cúpula vesical. A mucosa vesical presente ao redor do tumor pode se encontrar congesta e edemaciada.
Além disso, ainda na mucosa pode ser encontrado pequenos cistos transluscentes ou com aspecto azulado devido ao acúmulo de sangue.

Durante a menstruação: 0 tumor pode se apresentar com forma muito maior e mais congesto. As áreas císticas assumem aspecto azulado-enegrecido e menos translucente.

Após a menstruação: o tumor conserva sua área aumentada, mas a congestão e o edema são menos marcantes e o fluido enegrecido dos cistos ainda se mantém, mas com menor intensidade na coloração. Período intermenstrual: o tumor regride de tamanho e somente alguns cistos com fluido azulado podem ser vistos.

\section{Biópsia Transuretral}

Deve ser realizada em todos os casos onde há suspeita de endometriose vesical, pois ajudará a fazer diagnóstico diferencial com processos tumorais.

O material removido, caso seja superficial, pode apresentar diagnóstico de cistite inespecífica. Pode ser necessária ressecção com alça para permitir ao patologista uma quantidade suficiente de tecido com infiltração do epitélio endometrial.

\section{Estudo Anatomopatológico}

O aspecto histológico é necessário para ser feito o diagnóstico definitivo.

A princípio, identifica-se glândulas e/ou estroma endometrial aderido à parede vesical que, num estágio inicial, pode estar confinada à serosa e, se não for tratada, progride para a camada muscular, submucosa e mucosa ${ }^{20}$. A lesão endometrial pode ser desde única até múltipla, em forma de placas até massas císticas grandes. Recentemente foi descrita a presença de adenomiose invadindo a bexiga como na endometriose de septo retovaginal

À inspeção nota-se superfície irregular com fluido enegrecido proveniente dos cis-

Tabela 2 - Sinais associados
à endometriose vesical, em ordem
de incidência

tos que estão, na maioria das vezes, na superfície externa do tumor.

$\mathrm{Na}$ histologia encontra-se tecido endometrial infiltrado entre fibras musculares lisas da bexiga.

As glândulas podem estar preenchidas por sangue ou material necrótico. Raramente há degeneração maligna.

\section{Tratamento}

A escolha da terapêutica para endometriose vesical é da responsabilidade tanto do urologista quanto do ginecologista, e deve ser baseada em algumas condições como idade da paciente, desejo de gestações, extensão da lesão vesical, importância dos sintomas vesicais, presença de patologia pélvica e gravidade das alterações menstruais (tabela 4) ${ }^{22-28}$.

O tratamento da endometriose urinária 
deve ser individualizado. $\mathrm{O}$ acometimento da bexiga por adenomiose é freqüente $e$, nessa situação, a exerése total do nódulo endometriótico, seguida de laparoscopia, pode dispensar terapêutica hormonal complementar. A terapêutica medicamentosa deve ser aplicada nos casos onde não houve extirpação total da doença.

O tratamento clínico pretende criar um meio hormonal pouco favorável para a evolução dos implantes endometrióticos. As primeiras substâncias introduzidas mimetizavam a gestação, onde a anovulação é acompanhada de decidualização do endométrio com sua posterior atrofia.

A endometriose é afecção estrógeno dependente e, portanto, pode ser utilizado qualquer esquema terapêutico que diminua a ação deste hormônio.

$\mathrm{O}$ uso do acetato de medroxiprogesterona na dose de 50 a 100mg intramuscular mensal é suficiente para a remissão total da dor. Suas vantagens residem no seu baixo custo e na ausência de efeitos estrogênicos secundários. Os efeitos colaterais dependem da dosagem, da duração, do tratamento e da via de administração. As alterações menstruais são relatadas em 40 a $70 \%$ das usuárias. São também descritos náuseas, vômitos, aumento de peso, retenção hídrica, depressão e diminuição da libido nos quais constituem causas importantes de abandono do tratamento.

O danazol, desde sua introdução (197I), tem sido um dos fármacos mais utilizados na endometriose. É um derivado da 17-etiniltestosterona e seu mecanismo inclui um estado de hipoestrogenismo de origem central e periférica. A dose utilizada é de 400 a $800 \mathrm{mg}$ diários, induzindo amenorréia. Os efeitos colaterais mais comuns são aumento de peso, cãimbras, mudança de humor, pele oleosa, atrofia mamária e depressão.

gestrinona induz à redução dos receptores estrogênicos e progestagênicos

Tabela 4 - Opções terapêuticas utilizadas na abordagem da endometriose vesical
Tratamento clínico
Radioterapia
Tratamento cirúrgico

$$
\begin{aligned}
& \text { Dietibestrol } \\
& \text { Andrógenos } \\
& \text { Contraceptivo oral } \\
& \text { Danazol } \\
& \text { Análogos de GnRH }
\end{aligned}
$$

Ressecção e fulguraçãa

Cistectomia parcial, convencional

ou laparoscópica no endométrio, suprime o pico endógeno de LH e inibe a esteroidogênese ovariana. É utilizada na dose semanal de 5 a 7,5 mg. Pode causar alterações menstruais, queda de cabelo, acne e seborréia. Tanto o gestrinona quanto o danazol devem ser utilizados por seis meses.

Ultimamente, a utilização dos análogos do GnRH para o tratamento da endometriose tem aumentado. Seu uso prolongado causa supressão da função gonadotrófica através do mecanismo de down regulation nos receptores de $\mathrm{GnRH}$ da glândula pituitária. A falta da secreção de gonadotropinas e, especialmente, a carência da forma biológica ativa de LH e FSH irão resultar em estado hipoestrogênico ${ }^{22}$. São utilizados por várias vias de administração (intranasal, subcutânea, intramuscular). Nos tratamentos a longo prazo são preferíveis as formas de depósito. Os efeitos colaterais mais comuns são aqueles ocasionados por hipoestrogenismo. O tratamento além dos seis meses proporciona uma redução significativa da densidade óssea. Dessa forma, a maioria dos autores recomenda a terapia de reposição hormonal coadjuvante utilizando noretindrona ou I7-ß estradiol.

Stanley et al. apresentaram 10 casos de endometriose vesical dos quais sete foram submetidos à cistectomia parcial concomitante ao tratamento supressor de estró- genos, sendo que seis pacientes evoluíram bem. Os três pacientes restantes foram tratados com supressão hormonal apresentando bons resultados ${ }^{23}$. Lavelle et $a$. trataram clinicamente uma paciente com obstrução ureteral por endometriose e obtiveram sucesso ${ }^{24}$. Kistner demonstrou alívio dos sintomas com terapia progestagênica em mais de $80 \%$ das mulheres tratadas desta forma, entretanto Andrews acredita que esta terapêutica é paliativa e temporária ${ }^{25,26}$. Devido às desagradáveis conseqüências da supressão estrogênica, como osteoporose, por exemplo, Foster et al. acreditam que a melhor forma de abordar esta doença é através da cistectomia parcial, seguido de tratamento adjuvante com hormônios, se houverem implantes endometriais remanescentes ${ }^{27}$. Recentemente, Nezhat et al. descreveram 19 casos de sutura vesical, alguns após ressecção parcial de endometriomas por via laparoscópica. Estes pacientes foram mantidos com sonda vesical por 7- I 4 dias e apenas uma mulher apresentou fístula vésico-vaginal que necessitou de correção. Todos os outros casos evoluíram satisfatoriamente e o autor concluiu que 0 tratamento laparoscópico é factível ${ }^{28}$.

O tratamento de pacientes com endometriose é ainda motivo de discussão. Bons resultados foram obtidos com as várias formas terapêuticas e são defendidos por seus autores. Os casos devem ser analisados em 
TOBIAS M. ET AL.

suas particularidades como o tamanho da lesão, severidade dos sintomas, desejo reprodutivo da paciente e aderência ao tratamento.

A forma isolada de acometimento do trato urinário é rara, os ureteres podem estar comprometidos nas formas severas de endometriose pélvica. A bexiga é mais freqüentemente acometida por adenomiose, daí a importância da investigação endoscópica do trato genital superior.

As formas tumorais devem preferencialmente ser tratadas com a combinação de terapêutica cirúrgica e clínica sendo necessária a ressecção do tumor.

\section{RefERÊNCIAS Biblográficas}

I. Velebil P, Wingo PH, Xia, Z. Rate of hospitalization of gynecologic disorders among reproductive - age women in the United States. Obstet Gynecol 1995; 86: 764-7.

2. Redwine DB. The distribuion of endometriosis in the pelvis by age groups and fertility. Fertil Steril 1987; 47: 173-5.

3. Koninckx PR, Meuleman C, DemeyereS, Lesaffre E, Cornillie FJ. Suggestive evidence that pelvic endometriosis is a progressive disease, whereas deeply infiltrating endometriosis is associated with pelvic pain. Fertil Steril 1991; 55: 759-65.

4. Dmowski WP, Lesniewicz R, Rana N.,Pepping $P$, Noursalehi M. Changing trends in the diagnosis of endometriosis: a comparative study of women with pelvic endometriosis presenting with chronic pelvic pain or infertility. Fertil Steril 1997; 67: 238-43.

5. Sampson JÁ. Peritoneal endometriosis due to the menstrual dissemination of endometrial tissue into the peritonial cavity. Am J Obstet
Gynecol 1927; 14: 422-69.

6. Weed JC, Arguembourg PC. Endometriosis: can it produce an autoimune response resulting in infertility? Clin Obstet Gynecol 1980; 23: 885.

7. Badawy SZ, Cuenca V, Stitzel ,A, Jacobs RD B, Tomar RH. Autoimmune phenomena in infertile patients with endometriosis. Obstet Gynecol 1984; 63: 27I-5.

8. Ylikorkala $\bigcirc$, Tenhunen A. Folicular fluid prostaglandins in endometriosis and ovarian and hyperstimulation. Fertil Steril 1984; 41:66-9.

9. Bussaca M, Viganó P, Magri B, Natale A, Vignali $M$. Endometrial adhesium molecules and natural killer activity in the ethiopathogenesis of endometriosis. In: Coutinho, EM.. Spínola P, Moura LH. Progress in the management of endometriosis., 1994; London, The Parthenon Publishing Group. p | |-4.

10. DonnezJ, Nisolle M, Casanas-RouxF, Bassil S, Anaf $\checkmark$. Rectovaginal septum, endometriosis or adenomyosis: laparoscopic management in a series of 23 I patients. Human Reprod 1995; 10: 630-35.

I I. Donnez J, Nisolle M, Smoes P, Gillet N, Beguin S, Casanas-Roux F. Peritonial endometriosis and endometriotic nodules of the rectovaginal septum are two different entities. Fertil Steril 1996; 66: 362-8.

12. Peterson EP, Behrman SJ. Laparoscopy of infertile patient. J Obstet Gynecol 1970; 36: 363-7.

13. Goldenberg RL, Magendantz HG. Laparoscopy and infertility evaluation. Obstet Gynecol 1976; 47: 410-4.

14. Hasson HM. Incidence of endometriosis in diagnostic laparoscopy. J Reprod Med 1976; 16: 135-8.

15. Maluf M, Perin PM, Souza AZ, Izzo VM. Endometriose e esterilidade (considerações terapêuticas). Ginecol Obstet Bras 1986;9: 63-6.

16. Barbosa C P, Souza AMB, Silveira KF, Fukazawa EM, Cole APN. Achados endoscópicos em pacientes inférteis. In XVI Congresso Brasileiro de Reprodução Humana, São Paulo, 1994. Anais. São Paulo 1994; p. 160.

17. Mahmood TA, Templeton A. Prevalence and genesis of endometriosis. Hum Reprod 1991; 6: 544-9.

18. Liu DTY, Hitchcock A. Endometriosis: its as- sociation with retrograde menstruation, dysmenorrhoea and tubal pathology. Br J Obstet Gynaecol 1986; 93: 859-62.

19. Arap Neto W., Lopes RN, Cury M, Montelatto NID, Arap S. Vesical endometriosis. Urology $1984 ; 24$ (3):27| -4.

20. Abeshouse BS, Abeshouse G. Endometriosis of the urinary tract: a review of the literature and a report of four cases of vesical endometriosis. J Int Col Surg 1960; 34 (I): 43-58.

21. Vercellini P, Meschia M, De Giorgi O, Panazza S, Cortesi I, Crosignani G: Bladder detrusor endometriosis: clinical and pathogenetic implications. J Urol 1996 ; I 55:84-6.

22. Kiesel L, Runnebaum B. Neuroendocrine mechanisms of gonadotropin releasing hormone action. In: Brosens I, Jacobs HS, Runnenbaum B. LhRH Analogues in gynaecology. New Jersey, the Parthenon Publishing group LTD 1990; p 08.

23. Stanley KE.Jr, Utz D C, Dockerty M B. Clinically significant endometriosis of the urinaty tract. Surg Gynecol Obstet 1965; 120: 491-499.

24. Lavelle KJ, Melman AW, Clearly RE. Ureteral obstruction owing to endometriosis: reversa with progestin . J Urol 1976; | 1 6:665-668.

25. Kistner R. The use of progestins in obstetric and gynecology. In: Year book medical publishers. Chicago 1969 ;p 73.

26. Andrews WC. Medical versus surgical treatment of endometriosis. Clin Obstet Gynecol 1980; 23:917-922.

27. Foster RS, Riwk RC, Mulcahy JJ. Vesical endometriosis: Medical or surgical treatment. Urology 1987; 29 (1):64-65.

28. Nezhat CH, Seidman DS, NezhatF. Laparoscopic management of intentional and unintentional cistostomy. J Urol 1996; I 56: I 400- I 402.

29. Tobias-Machado M, Lopes Neto AC, Juliano RV, Simardi LH, Barbosa CP, Borelli M, Wroclawskier, Endometriose Simulando Neoplasia Vesical. Rev. Bras. Ginecol. Obstet. 2000(3): |4|- 446.

Artigo recebido: 14/01/2000

Aceito para publicação: 20/04/2000 